\title{
Density Matrix Approach to Local Hilbert Space Reduction
}

\author{
Chunli Zhang, Eric Jeckelmann, and Steven R. White \\ Department of Physics and Astronomy, University of California, Irvine, CA 92697
}

(May 7, 2018)

\begin{abstract}
We present a density matrix approach for treating systems with a large or infinite number of degrees of freedom per site with exact diagonalization or the density matrix renormalization group. The method is demonstrated on the 1D Holstein model of electrons coupled to Einstein phonons. In this system, two or three optimized phonon modes per site give results as accurate as with 10-100 bare phonon levels per site.

PACS Numbers: 74.20.Mn, 71.10.Fd, 71.10.Pm
\end{abstract}

During the past decade there have been great strides in the development of numerical techniques for simulating strongly correlated systems. A significant limitation to many of these methods - for example, exact diagonalization using the Lanczos algorithm, or the density matrix renormalization group [1] (DMRG) - is that they require a finite basis. In an electron-phonon lattice model, for example, the number of phonons is not conserved and the Hilbert space is infinite, even for a finite number of sites. Of course, the number of phonons can be artificially constrained, but for strongly coupled systems the number of phonons needed for an accurate treatment may be quite large. This often severely constrains the size of the systems which may be studied.

Here we present a technique for generating a controlled truncation of the Hilbert space, which allows the use of a very small local basis without significant loss of accuracy. The local basis which is generated can be used in exact diagonalization, DMRG, or other approaches to allow treatment of larger systems. The procedure is closely related to DMRG, in that the local basis is generated using a density matrix, but it is simpler to implement. We illustrate the method on the Holstein model, a model of noninteracting electrons on a lattice coupled to phonons, with one Einstein oscillator on each site. For this model we show that with two or three optimized phonon modes per site, we obtain the same accuracy as with dozens of unoptimized phonon levels per site. For simplicity, the Hilbert space reduction technique is used here in conjunction with exact diagonalization, but coupling it to other approaches, such as DMRG, would allow treatment of larger systems.

The key idea of this approach is identical to the key idea of DMRG [1]: in order to eliminate states from a part of a system without loss of accuracy, one should transform to the basis of eigenvectors of the reduced density matrix, and discard states with low probability. The key difference is that here the subsystem is a single site, or a handful of sites, rather than varying fractions of the entire system. To be specific, consider a many-body system divided into unit cells which we will call "sites", such that the complete Hilbert space of the system is the outer product of the states of the sites. The number of states per site may be finite or infinite. We will consider here only systems with translational invariance, so that all sites are equivalent. Let $i$ label the states of a particular single site, say site 1 . Let $j$ label the combined states of all of the rest of the sites. Then a wavefunction of the system can be written as

$$
|\psi\rangle=\sum_{i j} \psi_{i j}|i\rangle|j\rangle
$$

The density matrix for this site when the system is in the state $|\psi\rangle$ is

$$
\rho_{i i^{\prime}}=\sum_{j} \psi_{i j} \psi_{i j}^{*}
$$

Let $w_{\alpha}$ be the eigenvalues of $\rho$, and let $v_{\alpha}$ be the eigenvectors. The $w_{\alpha}$ are the probabilities of the states $v_{\alpha}$. If $w_{\alpha}$ is negligible, then the corresponding eigenvector $v_{\alpha}$ can be discarded from the basis for the site, without affecting the state $\psi$. If one wishes to keep a limited number of states $m$ per site, then the best states to keep are the eigenstates of $\rho$ with largest eigenvalues [1]. In the case of the Holstein model, we will show that all but a handful of these eigenstates have negligible probability.

Usually the target state $\psi$ which one wants to represent is the ground state. If one wants a site basis which represents several states, one can add each state into the density matrix

$$
\rho_{i i^{\prime}}=\sum_{\alpha} \sum_{j} a_{\alpha} \psi_{i j}^{\alpha} \psi_{i j}^{\alpha *},
$$

where the $a_{\alpha}$ are weights assigned to each target state, representing the importance of that state. Again, the optimal states to keep are the eigenstates of $\rho$.

Unfortunately, in order to obtain the optimal states, we need the target state, which we usually we do not know-usually we want the optimal states to help get the target state. This problem can be circumvented in several ways. We illustrate these approaches in the Holstein model, which has as Hamiltonian 


$$
\begin{aligned}
H= & \omega \sum_{\ell} b_{\ell}^{\dagger} b_{\ell}-\gamma \sum_{\ell}\left(b_{\ell}^{\dagger}+b_{\ell}\right) n_{\ell} \\
& -t \sum_{\ell \sigma}\left(c_{\ell+1 \sigma}^{\dagger} c_{\ell \sigma}+c_{\ell \sigma}^{\dagger} c_{\ell+1 \sigma}\right),
\end{aligned}
$$

where $c_{\ell \sigma}^{\dagger}$ and $c_{\ell \sigma}$ are electron creation and annihilation operators, $b_{\ell}^{\dagger}$ and $b_{\ell}$ are phonon creation and annihilation operators, $n_{\ell}=c_{\ell \uparrow}^{\dagger} c_{\ell \uparrow}+c_{\ell \downarrow}^{\dagger} c_{\ell \downarrow}$ and $t$ is the hopping integral, $\gamma$ is the electron-phonon coupling constant, and each oscillator has frequency $\omega$.

The first approach is illustrated in Fig. 1(a). Here one site of the system (the "big site") is allowed to have a large number of phonon states $M$, with $M \sim 10-100$. The rest of the sites have a much smaller number of phonon levels, $m \sim 2-3$. A set of Davidson or Lanczos exact diagonalizations are performed. In the first diagonalization, all of the phonon states are "bare": they are eigenstates of the single site phonon Hamiltonian, characterized by the frequency $\omega$. After each diagonalization, the density matrix for the phonon modes of the big site is diagonalized. The most probable $m$ eigenstates are the new optimal phonon modes. These optimal phonon modes are used on all the other sites for the next diagonalization. In each diagonalization, the big site always has a large number of phonon modes, so that it can generate improved optimal modes for the next iteration. The diagonalizations are repeated until the optimal modes have converged. In the first diagonalization, the optimal modes are not very accurate because the bare phonon levels used on the other sites severely truncate the Hilbert space. Convergence takes only a few diagonalizations, however.

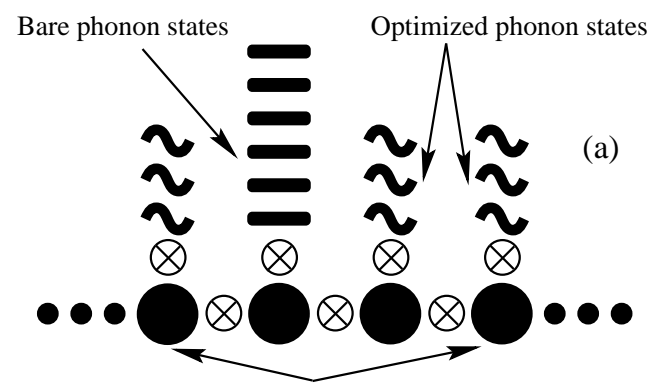

Fermion degrees of freedom on a site

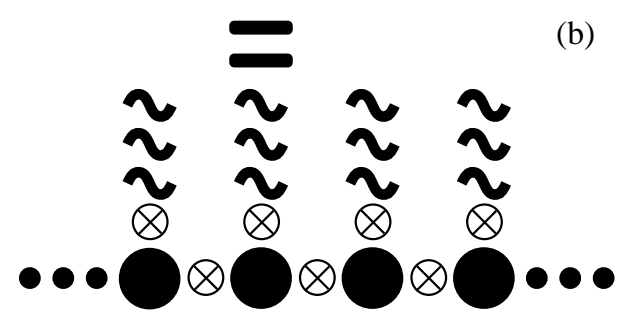

FIG. 1. Algorithms for constructing optimal bases. (a) The big site has the complete set of bare phonon levels. (b) The big site has the optimal levels plus a few bare levels.
While this approach is very simple, the large number of states on the big site limits the size of the system which can be diagonalized. A more sophisticated approach is illustrated in Fig. 1(b). Here, the big site has both the optimal modes and two or three extra levels, rather than $M$ bare levels. These extra levels allow improvement of the optimal basis. They are taken from the set of $M$ bare levels but are explicitly orthogonalized to the current optimal modes. After a diagonalization including these levels, a new density matrix is formed and optimal modes found. These optimal modes can mix in a little of the extra levels to improve the basis. The next diagonalization uses different extra levels. One sweep consists of enough diagonalizations to include all $M$ bare levels as extra levels. A couple of sweeps are needed to reach full convergence of the optimal levels. Each diagonalization uses as the starting wavefunction the converged wavefunction from the last step. Therefore only two or three Davidson steps are needed for convergence, rather than dozens.

A further improvement comes from forming the density matrix for an entire site, including electron degrees of freedom, rather than just the phonon levels. This forms different optimal phonon modes for each of the four electron states of the site, which reduces the number of states needed for a given accuracy.

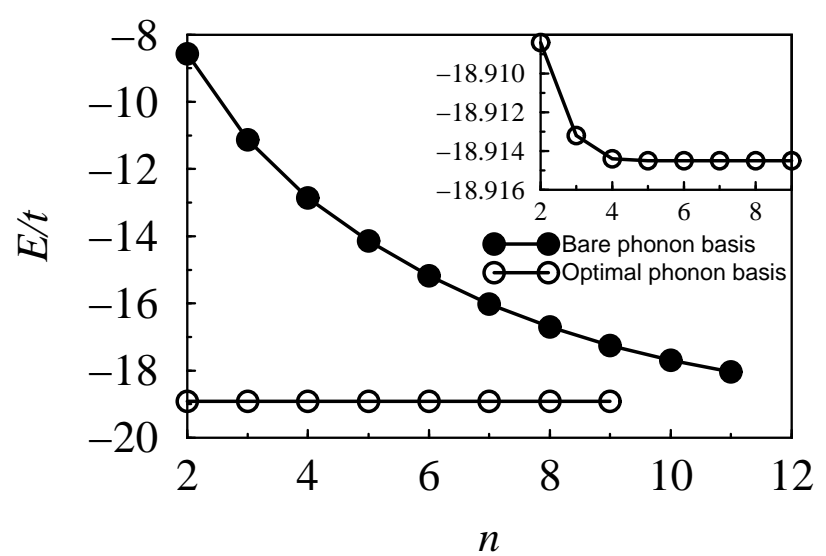

FIG. 2. Ground state energy of a 4-site half-filled Holstein system with $\omega=t$ and $\gamma=1.5 t$ as a function of the number of phonon states kept on each site of the lattice.

In Fig. 2 we show the ground state energy of a four site system as a function of the number of phonon modes, both for bare phonon levels and for optimal modes, where the $m$ optimal modes are allowed to be different for each electron state of a site. The energy in these restricted bases form upper bounds to the true ground state energy of the four site system. The improvement coming from using optimal modes is remarkable. Using only two optimal modes, the energy is accurate to less than $0.1 \%$, whereas keeping eleven bare modes (the largest number we could treat), the error is greater than $5 \%$. 
The very rapid convergence of the energy as a function of the number of optimal phonon modes is due to the very tight distribution of their probabilities $w_{\alpha}$. In two special cases $(\gamma=0$ or $t=0)$ it is possible to show that at most one optimal phonon level for each electron state of a site has a non-zero probability. For arbitrary couplings several optimal phonon levels can have a finite probability but only a few of them are significant. In the ground state of a 6 -site Holstein model with $\omega=t$, we have found that the third and fourth highest eigenvalues $w_{\alpha}$ (for a given electron state of a site) are smaller than $10^{-3}$ and $10^{-5}$, respectively, for any electron-phonon coupling $\gamma$. Therefore, we have never needed more than $m=2-3$ optimal phonon levels to get an accurate ground state.

In Fig. 3(a) we show the four most probable phonon states expressed in the bare phonon basis for the ground state of a 6 -site Holstein lattice. The probability $w_{\alpha}$ and the occupation of the electronic site are also given for each state. It is clear that one needs at least $\sim 20$ bare phonon levels for an accurate treatment of this system.

An additional improvement is often possible: the optimal levels may be transferable from a smaller system to a larger system. We find that in the Holstein model at half filling, the levels obtained from applying this procedure to a two site or four site system work very well for larger systems. Thus for the larger system, one needs to do only one diagonalization, and each site has only $m$ levels. This would be the simplest way to incorporate this approach into DMRG: use a small system diagonalization approach to find optimal levels, and then use DMRG to treat very long chains. Away from half-filling, because only certain fillings are possible on small systems, it would probably be necessary to simultaneously target two or more states with different fillings in order for the optimal basis to be transferable to a large system.

The form of the optimal phonon levels can tell us important information about the system. In Fig. 3(b) we show optimal phonon wavefunctions as a function of the oscillator position $q=b^{\dagger}+b$ for different electron-phonon couplings. Only the most probable optimized phonon level is shown for each possible electron state of a site. If the optimal states are allowed to be different for each electron state, we find that every optimal state is either an eigenstate of an oscillator with a shifted equilibrium position or a linear combination of two such eigenstates, to surprisingly high accuracy, with overlaps greater than $96 \%$. Unfortunately, we can not use this property to calculate a priori a basis of optimal states.

The form of the most important phonon states shown in Fig. 3(b) can be understood qualitatively in the weak and strong coupling regimes. The behavior at intermediate couplings interpolates smoothly between strong and weak coupling. In the weak coupling regime $(\gamma=0.5 t)$ optimal states are simply eigenstates of an oscillator with an equilibrium position $q \approx 2 \gamma / \omega$ as predicted by a mean-
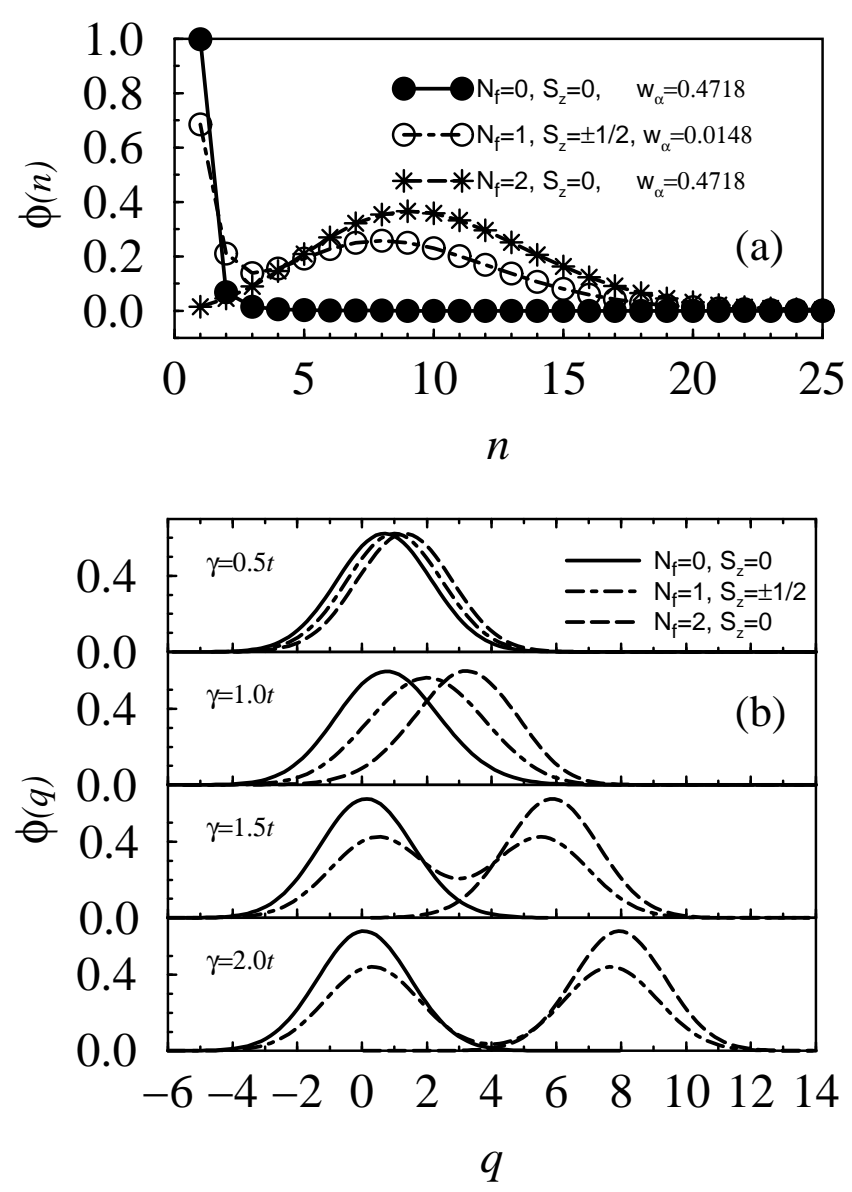

FIG. 3. (a) The first four optimal phonon states expressed in the bare phonon basis for a 6 -site half-filled Holstein system with $\omega=t$ and $\gamma=1.5 t$. (b) Optimal phonon wavefunctions as a function of the oscillator position $q$ for different electron-phonon couplings $\gamma$ for the same lattice.

field approximation. Differences between the optimal phonon states for the different electronic states of a site are small compared to their widths, which are determined by phonon fluctuations. Therefore, electron and phonons are almost independent and the lattice relaxation generated by the presence of electrons is much smaller than quantum lattice fluctuations.

In the strong coupling regime $(\gamma=2 t)$ we have found that the optimal states for $N_{f}=0,2$ are very similar to the ground states of oscillators with equilibrium positions $q \approx 2 N_{f} \gamma / \omega$. This corresponds to a bipolaronic ground state in which electrons are trapped by local lattice distortions and form pairs localized on a single site, with one pair for every two sites. Except for a small shift in the equilibrium positions, the optimal phonon states for a singly occupied site are almost the superposition of the optimal phonon states for $N_{f}=0,2$. This can be understood as a retardation effect: the $N_{f}=1$ states are intermediate states with low probability; electrons do not spend enough time in these states for the phonon states to adapt. These optimized phonon states for $N_{f}=1$ are 
very different from the phonon states generated by the Lang-Firsov transformation which shifts each oscillator equilibrium position by a quantity $2 N_{f} \gamma / \omega$. Therefore, it is not surprising that the standard strong coupling theory, which is based on the Lang-Firsov transformation, poorly describes the electronic and dynamical properties of the Holstein Hamiltonian [2].

Mean-field theory predicts that the ground state of the half-filled Holstein model is a charge-density-wave (CDW) state with a dimerized lattice and a gap at the Fermi surface for any finite electron-phonon coupling. An interesting question is whether the ground state is modified when quantum lattice fluctuations are taken into account. There is strong evidence that the system is dimerized for arbitrary finite coupling at finite phonon frequency [3, 国. On the other hand, recent results suggest that the gap is destroyed by quantum lattice fluctuations in the weak coupling regime [5]. In the 2 -site Holstein model it is known that there is a crossover from quasi-free electrons to a bipolaron at a finite coupling [2].

In Fig. 4 we present several quantities obtained with our method as a function of the electron-phonon coupling $\gamma$ for a 6 -site system with $\omega=t$. Our results for the phonon order parameter $m_{p}$, defined by $4 m_{p}^{2}=\left\langle\left(q_{\ell}-\right.\right.$ $\left.\left.q_{\ell+1}\right)^{2}\right\rangle-2$, where $q_{\ell}=b_{\ell}^{\dagger}+b_{\ell}$, are qualitatively similar to the predictions of previous studies [3,4]. Note that with this definition, $m_{p}=0$ for $\gamma=0$. We find $m_{p} \neq 0$ for any finite $\gamma$, although $m_{p}$ is smaller than the zeropoint lattice fluctuations in the weak coupling regime $\gamma \leq$ $0.8 t$. For $\gamma \geq 1.5 t, m_{p}$ approaches the strong-coupling theory result $m_{p}=2 \gamma / \omega$ The electronic static staggered susceptibility, defined as

$$
\chi_{f}=\sum_{\ell}(-1)^{\ell}\left\langle n_{i} n_{i+\ell}\right\rangle,
$$

indicates the existence of a transition around $\gamma=t$, where $\chi_{f}$ suddenly increases from the free electron result $\chi_{f}=1$ and approaches the value $\chi_{f}=6$ representative of a perfect CDW order in a 6 -site system (Fig. 4). Finally, in Fig. 4 we also show the next-nearest neighbor pairing correlation $\left\langle P_{\ell} P_{\ell+2}^{\dagger}\right\rangle$, where $P_{\ell}=c_{\ell \uparrow} c_{\ell \downarrow}$. This quantity has a peak around the value of $\gamma$ where the dimerization amplitude $m_{p}$ starts to dominate quantum lattice fluctuations and $\chi_{f}$ goes up rapidly.

In the weak coupling regime we have been able to study larger systems (with up to 40 sites) with a DMRG method using a different approach to handle the phonon Hilbert space [6]. We have found that despite the peak evident in Fig. 4, the pairing correlations $\left\langle P_{\ell} P_{\ell+m}^{\dagger}\right\rangle$ decrease as $1 / \mathrm{m}^{2}$, similar to the behavior of free electrons, even for a coupling as large as $\gamma=0.8 t$. In the strong coupling regime, pairing correlations decay exponentially because the dimerization opens a gap at the Fermi level [3]. Therefore, it is possible that there is a transition from a metallic to an insulating CDW phase at a finite electron-

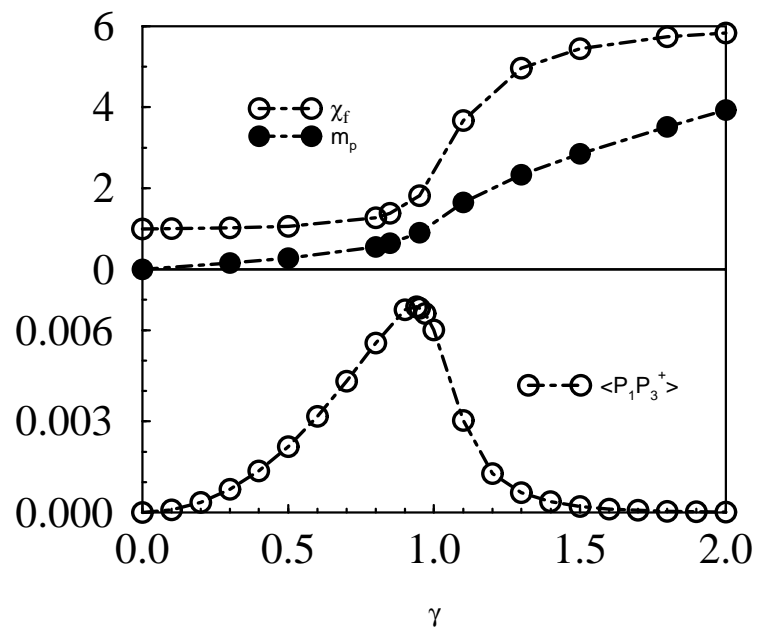

FIG. 4. Phonon order parameter $m_{p}$, staggered static electronic susceptibility $\chi_{f}$ and next-nearest neighbor pairing correlations $\left\langle P_{\ell} P_{\ell+2}^{\dagger}\right\rangle$ as a function of the electron-phonon coupling in a 6 -site Holstein lattice with $\omega=t$.

phonon coupling [5].

Obviously, a complete understanding of the Holstein model properties requires the study of larger systems. The use of optimized phonon basis coupled to powerful numerical methods, such as DMRG, will enable us to perform these calculations. Similarly, the techniques described in this letter could greatly improve our capability to perform numerical studies of other problems which involve an infinite Hilbert space.

We would like to thank J.T. Gammel and Alan Bishop for helpful discussions. SRW acknowledges support from the NSF under Grant No. DMR-9509945, and from the University of California through the Campus Laboratory Collaborations Program. EJ thanks the Swiss National Science Foundation for financial support. Calculations were performed at the San Diego Supercomputer Center.

[1] S.R. White, Phys. Rev. Lett. 69, 2863 (1992), Phys. Rev. B 48, 10345 (1993).

[2] J. Ranninger and U. Thibblin, Phys. Rev. B 45, 7730 (1992), E.V.L. deMello and J. Ranninger Phys. Rev. B 55, 14872 (1997).

[3] J.E. Hirsch and E. Fradkin, Phys. Rev. B 27, 4302 (1983).

[4] H. Zheng, D. Feinberg and M. Avignon, Phys. Rev. B 39, 9405 (1989).

[5] C.Q. Wu, Q.F. Huang and X. Sun, Phys. Rev. B 52, R15683 (1995).

[6] E. Jeckelmann and S.R. White, preprint (1997). 